\title{
Umsetzbarkeit und Erleben: Erste Erfahrungen mit einer Kurzform der anwendungsorientierten Parcoursprüfung im MSc-Studiengang Psychologie
}

\author{
Eva-Lotta Brakemeier ${ }^{a, b} \quad$ Kristin Pilz $^{a, c}$ Jan Richter ${ }^{a}$ Tim Kaiser ${ }^{a}$ \\ a Universität Greifswald, Greifswald, Deutschland; ${ }^{b}$ Philipps-Universität Marburg, Marburg, Deutschland; \\ 'Universitätsmedizin Greifswald, Greifswald, Deutschland
}

\section{Schlüsselwörter \\ Psychotherapeutengesetz · Parcoursprüfung • \\ Anwendungsorientierte Hochschullehre . \\ Approbationsstudium · Simulationspatientln}

\section{Zusammenfassung \\ Einleitung: Voraussetzung für die Approbation im Rahmen der neuen Psychotherapeutenausbildung ist u.a. das Beste- hen einer anwendungsorientierten Parcoursprüfung. Da diese Prüfung von Lehrenden und Studierenden als Heraus- forderung angesehen wird, wurde eine Kurzform der Par- coursprüfung im Rahmen einer Modulprüfung des MSc-Stu- diengangs Psychologie eingeführt und evaluiert. Methode: Für die 15-minütige Parcoursprüfung wurden 9 Kompe- tenzbereiche basierend auf praxisorientierten Psychothera- pieseminaren entwickelt, von denen 2 jeweils geprüft wur- den. Zur Standardisierung der Benotung wurde ein Bewer- tungsbogen konzipiert. Eine Mitarbeiterin erhielt ein Training als Simulationspatientin. Für die Evaluation wurde ein Fragebogen entwickelt, welcher u.a. die Umsetzbarkeit und das subjektive Erleben dieses Prüfungsformats aus der Sicht der an der Prüfung beteiligten Personen erfasst. Ergeb- nisse: 14 Prüfungskandidierende ließen sich durch 3 Prüfende prüfen. Die Gesamtdurchschnittsnote betrug 1,19 (Bereich: 1,0-2,3). Die Auswertung der Fragebögen zeigte, dass alle drei Gruppen die Prüfung als geeignet, die prak- tischen Fertigkeiten abzubilden, objektiv und praxisnah empfanden. Von den Prüfungskandidierenden gaben 50\%}

an, dass die Prüfung sie gestresst habe, während die Simulationspatientin sich nie und die Prüfenden sich gar nicht (78\%) oder kaum (22\%) gestresst fühlten. Diskussion: Die Ergebnisse dieser Pilotstudie weisen auf eine gute Umsetzbarkeit der Parcoursprüfung hin, wenngleich sie auch mit Stress für die Prüfungskandidierenden verbunden war. Die Pilotstudie wird limitiert durch eine kleine und wahrscheinlich verzerrte Stichprobe (motivierte Studierende) ohne Vergleichsgruppe. Abschließend werden die Vor- und Nachteile dieses Prüfungsmodells kritisch diskutiert.

(c) 2020 The Author(s)

Published by S. Karger AG, Basel

Feasibility and Experiences: First Practical

Knowledge with a Short Form of the Objective Structured Clinical Examination in the Master's Degree in Psychology

\section{Keywords}

Psychotherapist law - Objective structured clinical examination - Practice-oriented academic teaching • Approbation · Simulating patient

\section{Abstract}

Introduction: One of the requirements for the approbation as part of the new psychotherapist studies is, among other things, passing an objective structured clinical ex-
() 2020 The Author(s) Published by S. Karger AG, Basel Karger Open acces

This article is licensed under the Creative Commons Attribution 4.0 International License (CC BY) (http://www.karger.com/Services OpenAccessLicense). Usage, derivative works and distribution are permitted provided that proper credit is given to the author and the original publisher 
amination (OSCE) involving simulating patients. Since teachers and students regard this new format as a challenge, a short form of the OSCE was implemented in the module examination of the current master's degree in psychology and evaluated by a pilot study. Method: For the 15-min course examination, 9 areas of competences were developed based on practice-oriented psychotherapy seminars, 2 of which were tested. In order to standardize the grading, a standardized evaluation form was developed. A colleague received training to simulate a patient. For evaluation, a questionnaire was conceptualized which measured, among other things, the feasibility and subjective experiences of and during the new format as rated by the persons involved in the exam. Results: 14 examination candidates chose to be examined in this new format by 3 auditors. The overall averaged grade was 1.19 (range: 1.0-3). The evaluation of the questionnaire showed that all three groups rated the exam to be suitable for displaying practical skills, objective and close to the practical application. Of the examination candidates, $50 \%$ reported feelings of stress, while the simulating patient never and the auditors did not at all (78\%) or rarely felt stressed (22\%) during the different examinations. Discussion: Overall, the results of this pilot study indicate a good feasibility of the OSCE, although the examination candidates rated it to be stressful at the same time. The pilot study is limited by the small and probably biased sample (motivated students) without a comparison group. To conclude, pros and cons of this examination format will be critically discussed.

(c) 2020 The Author(s)

Published by S. Karger AG, Basel

\section{Einleitung}

Das Gesetz zur Reform der Psychotherapeutenausbildung vom 15. November 2019 [BGBl I, S. 1604] hat die Ausbildung, die zum Beruf des/der PsychotherapeutIn führt, grundlegend reformiert und auf eine neue Rechtsgrundlage gestellt. Anders als bisher wird die Approbation als PsychotherapeutIn künftig nicht mehr nach einer postgradualen Ausbildung erteilt, sondern nach dem erfolgreichen Abschluss eines Bachelor- und eines Masterstudiums sowie dem Bestehen der psychotherapeutischen Prüfung vergeben. Am 14. Februar 2020 hat der deutsche Bundesrat der für das Gesetz notwendigen und vom Bundesministerium für Gesundheit [2019] erlassenen Approbationsordnung für PsychotherapeutInnen (PsychThApprO) zugestimmt. Die finale Version der PsychThApprO wurde am 4. März 2020 veröffentlicht [Bundesgesetzblatt, 2020]. Durch diese Gesetzesänderung werden nicht nur neue Formate der praktischen Lehre in die Hochschullehre integriert, sondern sie erfordert gleichsam neue Prüfungsformate. Im Unterabschnitt
3 der Approbationsordnung wird die sogenannte anwendungsorientierte Parcoursprüfung als Teil der staatlichen Approbationsprüfung beschrieben [Bundesgesetzblatt, 2020]. Diese strukturierte klinisch-praktische Prüfung wurde nach Vorbild des internationalen Prüfungsformats "Objective Structured Clinical Examination" (OSCE) [Harden et al., 1975] entworfen, welches in der Medizin mittlerweile als anerkannter Standard zur Erfassung klinischer Kompetenzen gilt [Turner und Dankoski, 2008; Vaidya und Ramchandani, 2008; Chisnall et al., 2015]. Die medizinische OSCE-Prüfung beinhaltet verschiedene Stationen, welche die Prüfungskandidierenden in strukturierter Weise durchlaufen, um sowohl theoretisches als auch prozedurales Wissen zu testen. Die Bewertungen erfolgen durch eine standardisierte, vorher definierte Checkliste, um die Objektivität der Prüfung zu gewährleisten. Dieser bereits etablierte Ansatz für das Assessment klinischer Kompetenzen in der Medizin soll nun mittels der in der PsychThApprO beschriebenen Parcoursprüfung auch in der Psychologie zur Anwendung kommen. In der PsychThApprO wurde festgelegt, dass der praktische Parcours aus 5 Stationen (zu $30 \mathrm{~min}$ ) bestehen wird, bei denen jeweils geschulte SimulationspatientInnen eingesetzt werden. Die Prüfungskandidierenden durchlaufen die Stationen in Fünfergruppen. Dabei werden folgende Kompetenzbereiche der klinischpraktischen Tätigkeit geprüft [vgl. $\$ 48$, Absatz 2-6]:

1. PatientInnensicherheit: Die Prüfungskandidierenden haben zu zeigen, dass sie zu einer umfassenden Risikoeinschätzung in der Lage sind.

2. Therapeutische Beziehungsgestaltung: Die Prüfungskandidierenden haben zu zeigen, dass sie Probleme in der therapeutischen Beziehungsgestaltung erkennen und diesen Problemen in geeigneter Form begegnen.

3. Diagnostik: Die Prüfungskandidierenden haben zu zeigen, dass sie eine zutreffende psychotherapeutische Diagnose stellen.

4. Patienteninformation und Patientenaufklärung: Die Prüfungskandidierenden haben zu zeigen, dass sie durch angemessene Patienteninformation $\mathrm{zu}$ einer selbstbestimmten Patientenentscheidung beitragen.

5. Leitlinienorientierte Behandlungsempfehlungen: Die Prüfungskandidierenden haben zu zeigen, dass sie die PatientInnen angemessen und diagnosebezogen über empfohlene Behandlungsmöglichkeiten informieren und auch solche Behandlungsmöglichkeiten einbeziehen, die außerhalb des eigenen Spezialisierungsbereichs liegen.

In der PsychThApprO wird festgehalten, dass für jeden individuellen Prüfungstermin der anwendungsorientierten Parcoursprüfung ein Pool an Prüfungsaufgaben für die 5 genannten Kompetenzbereiche erstellt werden soll. Für jede Prüfungsaufgabe sind eine Beschreibung der Patientensituation, Angaben zu zugelassenen Hilfsmit- 
teln, Instruktionen für den Prüfenden, eine Rollenbeschreibung für den/die SimulationspatientIn und ein strukturierter Bewertungsbogen vorzulegen. Dieser strukturierte Bewertungsbogen sollte eine Musterlösung mit gewichteten Leistungsmerkmalen und eine Checkliste für jedes Leistungsmerkmal mit aufgabenspezifischen Einzelkriterien enthalten. Dabei sind für die jeweiligen Kriterien die höchstmöglich zu erreichende Punktzahl sowie die Bestehensgrenze in Prozent der insgesamt an der Station erreichbaren Punktzahl anzugeben. Ferner legt die PsychThApprO fest, dass die Prüfenden und die SimulationspatientInnen für die anwendungsorientierte Parcoursprüfung geschult werden müssen, wobei sich die Schulung auf die Kenntnisse und Fertigkeiten, die für eine ordnungsgemäße Durchführung und Bewertung der anwendungsorientierten Parcoursprüfung benötigt werden, erstrecken sollte. In diesen genannten Punkten orientiert sich die neue Parcoursprüfung an dem Vorbild der medizinischen OSCE-Prüfung, welche eine objektive, zuverlässige und valide Erfassung klinischer Kompetenzen gewährleisten soll. Diese Gütekriterien können durch eine sorgfältige Planung und Durchführung des Formates erfüllt werden [Chenot und Erhardt, 2003]. Entscheidend für die Reliabilität sind die Anzahl der Stationen, Konsistenz zwischen den Bewertungen der Prüfenden sowie das Festsetzen einer Bestehensgrenze [Chenot und Erhardt, 2003]. Diese Aspekte wurden bei der Konzeption der in der PsychThApprO beschriebenen Parcoursprüfung berücksichtigt (siehe oben). Ferner soll die Objektivität von OSCE-Prüfungen mittels Bewertungsbögen gesichert werden, was ebenfalls für die psychotherapeutische Parcoursprüfung festgelegt wurde. Schließlich weisen OSCEPrüfungen eine hohe Augenscheinvalidität auf [Chenot und Erhardt, 2003] - ein Anspruch, den die neue Parcoursprüfung ebenfalls erfüllen sollte.

Diese Prüfungsform wird von vielen in der Hochschullehre tätigen KollegInnen der klinischen Psychologie und Psychotherapie als eine große zukünftige Herausforderung angesehen [vgl. Wilhelm et al., in diesem Themenheft]. Im persönlichen Austausch über die zu erwartenden praktischen Prüfungsanteile wurde auch bei einem Teil der Studierenden ein großer Respekt bis Unbehagen spürbar. Auch wenn aktuelle politische Diskussionen nahe legen, dass die Durchführung der Approbationsprüfung an das Institut für medizinische und pharmazeutische Prüfungsfragen [IMPP, https://www.impp. de/start.html] delegiert werden könnte, wird es unabhängig von der Durchführung dieser praktischen Prüfungsformate für die Universitäten relevant sein, die Studierenden bereits während des zur Approbation führenden Masterstudiengangs "Klinische Psychologie und Psychotherapie" auf dieses neue Prüfungsformat vorzubereiten. Eine Möglichkeit wäre, bereits einzelne Module des Masterstudienganges durch vergleichbare anwendungsorien- tierte Formate zu prüfen. Dies bietet sich insbesondere für folgende Inhalte an, die in der PsychThApprO für den Masterstudiengang im Rahmen der hochschulischen Lehre festgehalten werden (vgl. Anlage 2 der PsychThApprO): spezielle Störungs- und Verfahrenslehre der Psychotherapie, angewandte Psychotherapie, vertiefte psychologische Diagnostik und Begutachtung.

Um erste Erfahrungen mit einem solchen neuen Prüfungsformat zu gewinnen, wurde den Studierenden des bestehenden Studienganges MSc Psychologie an der Universität Greifswald im Wintersemester 2019/2020 für das Modul "Klinische Psychologie und Psychotherapie" erstmalig eine Kurzversion der anwendungsorientierten Parcoursprüfung als Alternative zur klassischen mündlichen Prüfung angeboten. Dabei verfolgten wir das Ziel zu prüfen, inwiefern sich die im Gesetz formulierten Anforderungen an die anwendungsorientierte Parcoursprüfung in einem Kurzformat als Modulprüfung in einem MSc-Studiengang Psychologie umsetzen lassen. Hierfür evaluierten wir das Prüfungsformat und untersuchten systematisch, wie die Prüfungskandidierenden, das prüfende Lehrpersonal und die Simulationspatientin diese Form der Prüfung erlebten und deren Durchführbarkeit beurteilten.

\section{Beschreibung der entwickelten Kurzversion einer Parcoursprüfung}

Im Modul B "Klinische Psychologie und Psychotherapie" des bestehenden MSc-Studiengangs Psychologie an der Universität Greifswald wurden im vergangenen Wintersemester 2019/20 vier praxisnahe klinische Seminare angeboten. Aufgrund der implementierten Lehre zu psychotherapeutischen Praktiken eignete sich diese Modulprüfung dazu, erstmalig das neue Prüfungsformat anzubieten. In der Tabelle 1 werden die 4 Seminare kurz beschrieben, welche die Basis für die Prüfungsinhalte bildeten.

Diese 4 Seminare verfolgten somit bereits die im Paragraph 1 Abschnitt 3 der PsychThApprO geforderte Vermittlung des theoretischen Wissens und die Entwicklung von therapeutischen Kompetenzen unter Beachtung von Patientensicherheit und Patientenrechten.

Da nach einer kurzfristigen Ankündigung des neuen Prüfungsformates die Mehrheit der Studierenden zunächst Misstrauen und Bedenken gegenüber der neuen Prüfungsform äußerten, entschieden wir, das neue Prüfungsformat als ergänzende Alternative zu der bisher durchgeführten traditionellen mündlichen Prüfung anzubieten. In dieser klassischen mündlichen Prüfung werden psychotherapeutisches Fakten- und Handlungswissen abgeprüft sowie vertiefenden Fragen zur Psychotherapie gestellt. Das gleichzeitige Abnehmen der beiden Formate in einer Prüfungsperiode hätte in unserer Evaluation einen interessanten Vergleich des traditionellen 
Tabelle 1. Beschreibung der 4 Seminare, welche die Basis für die anwendungsorientierte Parcoursprüfung bildeten (weitere Informationen finden sich hier: https://psychologie.uni-greifswald.de/storages/uni-greifswald/fakultaet/mnf/psychologie/vorlesungsverz/ KVV_MSc_WS1920_-_081019.pdf)

Titel Beschreibung

Das "Unified Protocol": Inhalt: Die klinisch-psychologische Forschung hat in den letzten Jahren herausgearbeitet, dass zahlreichen transdiagnostische möglichen Diagnosen einige wenige pathologische Prozesse zugrunde liegen. Dieser Erkenntnis folgend Behandlung emotionaler Störungen verschiedener psychischer Störungen gleichermaßen behandeln. In diesem Seminar wurde ein neuartiges transdiagnostisches Verfahren vorgestellt und eingeübt: das Unified Protocol. Dieses Verfahren ermöglicht durch acht verschiedene Behandlungsmodule, welche aus der kognitiven Verhaltenstherapie stammen, die Therapie verschiedener emotionaler Störungen (wie Angst- und Panikstörungen, depressive Störungen, Zwangsstörungen sowie Traumafolgestörungen)

Didaktik: Live-Demonstrationen, Videobeispiele, paarweise Rollenspiele an Fallbeispielen und eigenen Problemen

Basis-Literatur: Barlow und Farchione [2018]

Videobeispiele: https://www.youtube.com/channel/UC3Q9uoV1XPVzY_Q9cUgiiTg

Zwangsstörungen Inhalt: Das Seminar stellt die Symptomatik, Klassifikation und Diagnostik von Zwangsstörungen, neurobiologische und psychologische Modelle zur Entstehung und Aufrechterhaltung sowie unterschiedliche Ansätze zur Behandlung von Zwangsstörungen vor. Neben kognitiv-verhaltenstherapeutischen Interventionen werden ebenso neuere psychotherapeutische Ansätze der so genannten 3. Welle (z.B. metakognitive Therapie) und medikamentöse Therapien erarbeitet sowie deren differentielle Wirksamkeit anhand aktueller Studien kritisch diskutiert

Didaktik: Unter Einbezug vielfältiger didaktischer und interaktiver Methoden (z.B. Fallbeispiele, Videosequenzen, Rollenspiele, Journal-Clubs, Impulsreferate) sollen die Wissensinhalte der Veranstaltung mit Praxisbezug anschaulich vermittelt werden

Basis-Literatur: Wahl-Kordon et al. [2019]

Moderne Ansätze der Psychotherapie in der Praxis

Verhaltenstherapeutisches Fallseminar
Inhalt: Nach einem Überblick über die Vielfältigkeit der modernen Ansätze und aktuellen Entwicklungen werden wir im Seminar folgende vier moderne und evidenzbasierte Psychotherapiemethoden intensiver im direkten Vergleich betrachten: das Cognitive Behavioral Analysis System of Psychotherapy (CBASP), die dialektisch-behaviorale Therapie (DBT), die mentalisierungsbasierte Therapie (MBT) und die Schematherapie. Diese Psychotherapien vereint, dass sie alle die Erlebnisse aus der Kindheit als zentral für die Entstehung von psychischen Störungen ansehen und sich als integrative Methoden verstehen. In der konkreten Umsetzung unterscheiden sie sich jedoch teilweise erheblich, was u.a. durch Videos von derselben interaktionell schwierigen Psychotherapiesituation gezeigt wird. Durch diese konkreten Vergleiche adressieren wir auch die u.a. von Alan E. Kazdin mehrfach aufgeworfene wichtige Psychotherapiefrage: "What works for whom?" - d.h., welche Methode passt am besten zu welchem individuellen Patienten? Aber auch: Welche Therapierichtung passt am besten zu meiner eigenen (zukünftigen) therapeutischen Haltung und Identität?

Didaktik: Unter Einbezug vielfältiger didaktischer und interaktiver Methoden (z.B. Videosequenzen, Fallbeispiele, Rollenspiele, Übungen) soll neben den Wissensinhalten der Veranstaltung insbesondere ein praktischer Einblick in die modernen Psychotherapiemethoden gegeben werden

Basisliteratur: Brakemeier [2019]

Inhalt: Grundlage einer Planung und Durchführung einer zielführenden kognitiv-verhaltenstherapeutischen Intervention ist die Erstellung eines individuellen Störungsmodelles unter der Berücksichtigung prädisponierender, auslösender und aufrechterhaltender Faktoren. Besondere Berücksichtigung finden dabei die vertikalen und horizontalen Verhaltensanalysen. Ziel dieses Seminars ist es, an zur Verfügung gestellten Patientenbeispielen, selbstständig individuelle Störungsmodelle zu erarbeiten und eine begründete Therapieplanung abzuleiten

Didaktik: Nach einer theoretischen Einführung in die Thematik werden die Beschwerdebilder von mehreren Patienten anhand von kurzen Falldokumentationen oder Videos aufgezeichneter Erstgespräche vorgestellt. Unter Berücksichtigung von selbsterfahrungsbezogenen Übungen, angeleiteten Aufgaben und auswertenden Gruppendiskussionen werden Einzelschritte in der Erstellung eines individuellen Störungsmodelles geschult. Schließlich erarbeiten die teilnehmenden Studierenden selbstständig eine vollständige Fallkonzeption mit einer zu begründenden Behandlungsplanung

Basisliteratur: Kanfer et al. [2012] 
Tabelle 2. Liste der 9 zu prüfenden Kompetenzbereiche aus der Parcoursprüfung und die zugrunde liegenden Seminare des Moduls

\begin{tabular}{lll}
\hline $\begin{array}{l}\text { Kompetenz- } \\
\text { bereich }\end{array}$ & Basis-Seminar & Titel \\
\hline 1 & $\begin{array}{l}\text { Vermittlung des Störungsmodells und Ableitung des Therapierationales (insb. } \\
\text { Exposition) }\end{array}$ & Zwangsstörungen \\
\hline 2 & $\begin{array}{l}\text { Nichtbewertende, gegenwartsbezogene Aufmerksamkeit (Achtsamkeit bei } \\
\text { emotionalen Erfahrungen) }\end{array}$ & $\begin{array}{l}\text { Unified Protocol (Modul 3 bzw. } \\
\text { erster Core Skill) }\end{array}$ \\
\hline 3 & Kognitive Bewertung und Umbewertung & $\begin{array}{l}\text { Unified Protocol (Modul 4 bzw. } \\
\text { zweiter Core Skill) }\end{array}$ \\
\hline 4 & Problematische Handlungstendenzen erkennen und verändern & $\begin{array}{l}\text { Unified Protocol (Modul 5 bzw. } \\
\text { dritter Core Skill) }\end{array}$ \\
\hline 5 & $\begin{array}{l}\text { Bewusstsein für und Toleranz gegenüber körperlichen Empfindungen durch } \\
\text { interozeptive Expositionsübungen }\end{array}$ & $\begin{array}{l}\text { Unified Protocol (Modul 6 bzw. } \\
\text { vierter Core Skill) }\end{array}$ \\
\hline 6 & Konfrontation und Exposition (in sensu und in vivo) & $\begin{array}{l}\text { Unified Protocol (Modul 7), } \\
\text { Zwangsstörungen }\end{array}$ \\
\hline 7 & $\begin{array}{l}\text { Umgang mit schwierigen interpersonellen Situationen während der Sitzung durch } \\
\text { die Anwendung von CBASP-Strategien }\end{array}$ & Moderne Ansätze \\
\hline 8 & $\begin{array}{l}\text { Umgang mit schwierigen interpersonellen Situationen während der Sitzung durch } \\
\text { die Anwendung von DBT-Strategien }\end{array}$ & Moderne Ansätze \\
\hline 9 & $\begin{array}{l}\text { Umgang mit schwierigen interpersonellen Situationen während der Sitzung durch } \\
\text { die Anwendung von Strategien der Schematherapie }\end{array}$ & Moderne Ansätze
\end{tabular}

CBASP, Cognitive Behavioral Analysis System of Psychotherapy; DBT, dialektisch-behaviorale Therapie.

mit dem neuen Format ermöglicht. Da sich letztlich von 16 Prüfungskandidierenden nur 2 für das traditionelle Format entschieden, welche sich beide für die Prüfung krank meldeten, fand keine Prüfung im klassischen Format statt, sodass auch der Vergleich entfiel.

Zum Zwecke der Vorbereitung und standardisierten Durchführung der Modulprüfung wurde ein Informationsblatt erstellt (Online-Supplementmaterial 1; siehe www.karger.com/doi/10.1159/000507820), welches u.a. die praktischen Prüfungsaufgaben für die zu prüfenden 9 Kompetenzbereiche enthält. Dieses Dokument wurde auch dem Leiter des Prüfungsamtes übermittelt, welcher aufgrund der eingeräumten Wahlmöglichkeit zwischen der traditionellen und neuen Prüfung keine Bedenken sah. Im Kasten 1 ist das neue Prüfungsformat, welches aus zwei Prüfungsteilen bestand, kurz skizziert.

\section{Kasten 1: Kurzbeschreibung des neuen}

Prüfungsformats

Teil 1: Mündlich-praktische Fallprüfung (10 min)

Während dieses Prüfungsteils wurde psychotherapeutisches Fakten- und Handlungswissen geprüft. Die zu prüfende Person hatte fallspezifische Fragen zu der/dem als Prüfungsfall ausgewählten PatientIn oder typischen Fallbeispielen aus den Seminaren zu beantworten.
Teil 2: Anwendungsorientierte Parcoursprüfung (15 $\min$ )

Die anwendungsorientierte Parcoursprüfung wurde mit Hilfe von einer Schauspielpatientin durchgeführt, die zuvor für die Prüfung geschult worden war. Sie spielte jeweils PatientInnen, welche die typische, in den Seminaren behandelte Symptomatik aufwiesen. In der Tabelle 2 finden sich die 9 Kompetenzbereiche, in denen die Prüfung erfolgen konnte. Ihre Auswahl beruhte auf den Kompetenzbereichen, welche in den 4 Basis-Seminaren vorgestellt und geübt worden waren. Die oder der Studierende konnte vor der Prüfung der oder dem 1. PrüferIn 3 Kompetenzbereiche angeben, welche mindestens 2 der 4 Seminare abdecken sollten. Von diesen 3 Kompetenzbereichen wurden durch die Prüfenden 1-2 Bereiche zur Prüfung ausgewählt.

Zur Durchführung der Kompetenzbereiche 2-5 standen in der Prüfung die benötigten Arbeitsblätter zur Verfügung, die von den Prüfungskandidierenden verwendet werden sollten. Bei den Kompetenzbereichen 7-9 konnten folgende 4 schwierige interpersonelle Situationen in der Prüfung vorkommen, basierend auf den Patientenbeispielen, welche im Seminar behandelt wurden [vgl. Brakemeier, 2019]. Die Prüfungskandidierenden konnten bis 1 Woche vor der Prüfung zwei Situationen auswählen, von denen eine in der Prüfung dargestellt wurde: 
- Der wütende Patient basierend auf dem DVD-Patientenbeispiel "Leo Kroll"

- Der verschlossene Patient basierend auf dem DVDPatientenbeispiel "Lasse Sander"

- Der suizidale Patient basierend auf dem DVD-Patientenbeispiel "Peter Brauer"

- Der verliebte Patient basierend auf dem DVD-Patientenbeispiel "Fritz Wohlfahrt"

\section{Evaluation}

Um zu untersuchen, wie die Prüfungskandidierenden, die Prüfenden und die Simulationspatientin diese Art der Prüfung erlebten und deren Umsetzbarkeit beurteilten, entwickelten wir einen Evaluationsfragebogen. Da unseres Wissens nach kein validierter, allgemein anerkannter Fragebogen zur Evaluation derartiger Parcoursprüfungen vorliegt, haben wir bewährte Items eines Fragebogens, der bei OSCE-Evaluationen der Universitätsmedizin Greifswald eingesetzt wird, übernommen und weitere uns interessierende Items hinzugefügt. Der daraus resultierende "Fragebogen zur Evaluation einer Kurzform der anwendungsorientierten Parcoursprüfung" (Akronym: FEKaP) liegt in 3 Versionen vor, da uns die Sicht der Prüfungskandidierenden (FEKaP-Pk), der Prüfenden (FEKaP-P) sowie der/des SimulationspatientIn (FEKaP-S) in jeder Prüfung interessierte.

Ein Item des FEKaP-Pk lautet beispielsweise: "Die Prüfung war geeignet, meine erworbenen praktischen Fertigkeiten aus den Seminaren abzubilden.” Die Items konnten auf einer 5-stufigen Likert-Skala beantwortet werden (von 1 "stimme nicht zu" bis 5 "stimme zu").

Alle drei Varianten des Fragebogens befinden sich im Online-Supplementmaterial 2.

Die Prüfungskandidierenden wurden jeweils nach ihrer Prüfung und Notenbekanntgabe über die Evaluation informiert und um Teilnahme gebeten. Dabei wurde ihnen versichert, dass die Evaluation auf freiwilliger Basis erfolgt und anonymisiert ausgewertet wird (vgl. Seite 1 des FEKaP-S). Die Prüfenden und die Simulationspatientin waren durch die Entwicklung mit der Evaluation vertraut. Die Studie wurde gemäß der allgemeinen ethischen Richtlinien (z.B. nach der Deklaration von Helsinki) durchgeführt.

\section{Durchführung der Parcoursprüfungen}

$\mathrm{Zu}$ den Prüfungen, welche am 11. und 12. Februar 2020 im psychologischen Institut der Universität Greifswald stattfanden, meldeten sich 18 Studierende an. Von diesen Studierenden erschienen $14 \mathrm{zu}$ den Prüfungen, da es 4 Krankmeldungen gab, von denen 2 die traditionelle Variante gewählt hatten. Alle 14 Prüfungskandidieren- den, die zur Prüfung erschienen, hatten die neue Prüfungsform gewählt. Die Prüfungen wurden jeweils in wechselnden Paaren von den Autoren E.-L.B., T.K. und J.R. durchgeführt, wobei eine bzw. einer von beiden den Prüfungsvorsitz innehatte. Die Autorin K.P. übernahm die Rolle der Simulationspatientin in allen Prüfungen.

Für jede Prüfung wurden 30 min eingeplant, welche wie folgt aufgeteilt werden sollten: $10 \mathrm{~min}$ Teil 1, $15 \mathrm{~min}$ Teil 2 sowie 5 min Nachbesprechung der Prüfenden einschließlich Mitteilung der Note an den geprüften Studierenden.

\section{Bewertung}

Um eine möglichst standardisierte und objektive Bewertung und Benotung vornehmen zu können, wurden für jeden zu prüfenden praktischen Kompetenzbereich zuvor ein ähnlich der in der PsychThApprO beschriebener strukturierter Bewertungsbogen konzipiert, welcher eine Musterlösung mit gewichteten Leistungsmerkmalen, eine sich daraus ergebende Checkliste für jedes Leistungsmerkmal mit aufgabenspezifischen Einzelkriterien sowie die für jedes Leistungsmerkmal höchstmögliche Punktzahl enthält. Die Tabelle 3 gibt Ausschnitte dieser Bewertungsbögen wieder. In gesamtem Umfang befinden sie sich im Online-Supplementmaterial 3.

\section{Vorbereitung der Prüfenden}

Da die Prüfenden (E.-L.B., T.K., J.R.) auch bei 3 der 4 Basisseminare selbst die Lehrenden waren, war mindestens eine bzw. einer mit den Kompetenzbereichen ausführlich vertraut. Die oder der jeweils andere Prüfende wurde durch Lektüre der Seminarunterlagen, einer mündlichen Erläuterung der Kompetenzbereiche durch den Lehrenden sowie die Bewertungsbögen auf die Prüfung der jeweils anderen Kompetenzbereiche vorbereitet. Der Lehrende des Seminars Zwangsstörungen konnte bei der Prüfung nicht anwesend sein und übergab daher die Seminarinhalte durch das Übersenden des Seminarablaufs und schickte Prüfungsfragen für den Teil 1 der Prüfung. Die in diesem Seminar vermittelte Basistechnik der Verhaltenstherapie - Exposition mit Reaktionsverhinderung - wurde vergleichbar unterrichtet wie im Seminar zum Unified Protocol. Daher konnte auch die Prüfung des in diesem Seminar gelehrten Kompetenzbereiches durch die anderen Lehrenden durchgeführt werden.

\section{Vorbereitung der Simulationspatientin}

Aufgrund der überschaubaren Anzahl an Prüfungsanmeldungen und des Ziels der möglichst hohen Standardisierung wurde nur eine Simulationspatientin 
Tabelle 3. Strukturierter Bewertungsbogen mit Leistungskriterien von ausgewählten Kompetenzbereichen

\begin{tabular}{|c|c|}
\hline Kompetenzbereich & Kriterien (max. Punktzahl) \\
\hline \multicolumn{2}{|c|}{ Kompetenzbereiche der Seminare Unified Protocol und Zwangsstörungen } \\
\hline \multirow{5}{*}{2 Achtsamkeit } & Vermittlung von Inhalten $(3)$ \\
\hline & - Objektive, nichtwertende Haltung gegenüber Emotionen, gegenwartsbezogene Aufmerksamkeit \\
\hline & Durchführung (geführte Meditation oder Emotionsinduktion mit Musik) (5) \\
\hline & - Durchführung \\
\hline & Nachbesprechung (2) \\
\hline \multirow[t]{4}{*}{3 Kognitive Flexibilität } & Vermittlung von Inhalten (3) \\
\hline & - Wechselseitige Beziehung Gedanken und Gefühle, automatische Gedanken, Denkfallen \\
\hline & Durchführung (mehrdeutiges Bild, Pfeil-Übung oder Denkfallen - 1-2 Übungen) (5) \\
\hline & Nachbesprechung (2) \\
\hline \multirow[t]{3}{*}{5 Körperliche Empfindungen } & Einführung (3) \\
\hline & - Klärung evtl. medizinischer Kontraindikationen \\
\hline & $\begin{array}{l}\text { Durchführung (interozeptive Exposition nach Vorgabe, maximal } 2 \text { Übungen) (5) } \\
\text { - Nachbesprechung (2) }\end{array}$ \\
\hline \multirow{6}{*}{6 Exposition $^{1}$} & Einführung (2) \\
\hline & - Alte vs. neue Assoziationen, Gründe für Exposition \\
\hline & Erarbeitung einer Expositionshierarchie \\
\hline & Vorbereitung (u.V. Arbeitsblatt) (2) \\
\hline & Durchführung Exposition (5) \\
\hline & Nachbesprechung (u.V. Arbeitsblatt) (1) \\
\hline \multirow{2}{*}{$\begin{array}{l}\text { Übergreifende Kriterien für } \\
\text { die Kompetenzbereiche } 2-6\end{array}$} & Adäquate Reaktion auf Widerstand, Vermeidung und sonstige Probleme (3) \\
\hline & Beachtung von Grundregeln der Gesprächsführung (2) \\
\hline \multicolumn{2}{|c|}{ Kompetenzbereiche des Seminars Moderne Ansätze } \\
\hline \multirow{9}{*}{$\begin{array}{l}7 \text { CBASP (verschlossen, } \\
\text { suizidal, wütend, verliebt) }\end{array}$} & Einsatz des disziplinierten persönlichen Einlassens (4) \\
\hline & - Situation fürs disziplinierte persönliche Einlassen wahrnehmen, durch Empathietraining Patient \\
\hline & unterbrechen, Selbstoffenbarung diszipliniert vornehmen, zum “interpersonellen Problem” werden, \\
\hline & $\begin{array}{l}\text { Bezug zu den Pragungen herstellen, in die interpersonelle Diskriminationsubung uberleiten } \\
\text { Anwendung der interpersonellen Diskriminationsübung (3) }\end{array}$ \\
\hline & - Vergleich der Reaktion von früheren Bezugspersonen mit der eigenen Reaktion, Unterschiede \\
\hline & $\begin{array}{l}\text { benennen lassen und nach Bedeutung/Emotionen fragen } \\
\text { Qualität der therapeutischen Beziehungsgestaltung bzw. - bei Indikation - }\end{array}$ \\
\hline & Anwendung weiterer CBASP-Strategien (3) \\
\hline & - Sicherheitszone, Empathie und Wärme, effektives Zuhören, angemessene Kontrolle der \\
\hline & $\begin{array}{l}\text { Sitzung,Toleranz des negativen Affektes des Patienten, Einsatz weiterer Techniken wie Situations- } \\
\text { analyse und Kiesler-Kreis bei Indikation }\end{array}$ \\
\hline
\end{tabular}

8 DBT (verschlossen, suizidal, Anwendung von DBT-spezifischen Techniken (4)

wütend, verliebt) - - Abfragen der Arbeitsfähigkeit, Skillsübungen einbauen, Abfrage Diary Card, Fokus auf Exploration der (primären) Gefühle, Blockieren von dysfunktionalem Verhalten, Beziehungsmuster benennen, Verhaltensanalysen

Fokus auf Emotionen (3)

- Zusammenarbeit, Empathie, effektives Zuhören, angemessene Kontrolle der Sitzung, Toleranz des negativen Affektes des Patienten, Einsatz weiterer Techniken wie Verhaltensanalysen anwenden

Allgemeine DBT-Haltung/therapeutische Beziehungsgestaltung (3)

- Validierung, Akzeptanz, Authentizität, DBT-Wippe, Grenzen einhalten für sich und Patienten, direktives Vorgehen

Übergreifend für die

Kompetenzbereiche 7-9

Adäquate Reaktion auf Widerstand, Vermeidung und sonstige Probleme (3)

Beachtung von Grundregeln der Gesprächsführung (2)

CBASP, Cognitive Behavioral Analysis System of Psychotherapy; DBT, dialektisch-behaviorale Therapie. ${ }^{1}$ Bei der Wahl des Kompetenzbereiches Exposition wurde auf die Prüfung eines zweiten Kompetenzbereiches verzichtet, da die Prüfung des Kompetenzbereiches die gesamte Prüfungszeit in Anspruch nahm. 
(K.P.) für diese Prüfung trainiert. Dabei handelt es sich um eine Psychologin in Ausbildung zur Psychologischen Psychotherapeutin, welche im Rahmen ihrer wissenschaftlichen Tätigkeit an der Universitätsmedizin Greifswald bereits zuvor Erfahrungen mit Prüfungen nach dem Format der OSCE von Studierenden der Medizin sowohl in der Rolle der Simulationspatientin als auch der Prüfenden gesammelt hat. Zudem war sie als ehemalige Studentin im Fach Psychologie an der Universität Greifswald mit den vorherigen Formaten sowie der dortigen Lehre vertraut. Hierdurch konnte sie - in Absprache mit den Prüfenden - standardisierte und dem von den Prüfungskandidierenden geforderten Leistungsstand entsprechende Fälle entwickeln, welche sie in der Prüfung schauspielerte. Ihre Vorbereitung auf die Prüfungen bestand darin, dass sie die vorhandenen Lehrvideos, Arbeitsblätter und Patientenfälle aus der angegebenen Prüfungsliteratur studierte und auf Basis dessen ihre geschauspielerten Fälle entwickelte. Des Weiteren nahm sie an einer von den Prüfenden durchgeführten Schulung teil, bei welcher sie in den einzelnen Kompetenzbereichen (v.a. durch Rollenspiele) trainiert wurde. Hierbei ist darauf geachtet worden, dass für jeden Kompetenzbereich bestimmte Verhaltensweisen des/der PatientIn festgelegt wurden, anhand dessen die Prüfungskandidierenden entsprechend der Musterlösung bewertet werden konnten.

\section{Wahl der Kompetenzbereiche}

Die Kompetenzbereiche 2, 3, 5 und 7 wurden von den Prüfungskandidierenden am häufigsten gewählt, die beiden Kompetenzbereiche 1 und 9 wurden nie gewählt. Bei der Auswahl der Kompetenzbereiche durch die Prüfenden wurde darauf geachtet, dass möglichst alle gewählten Kompetenzbereiche abwechselnd geprüft wurden, sodass in den 14 Prüfungen entsprechend alle Bereiche bis auf 1 und 9 auch geprüft wurden.

\section{Benotung}

Die Prüfenden waren angehalten, Teil 1 und Teil 2 der Prüfungen separat zu benoten, wobei der Teil 2 aufgrund der zeitlichen Schwerpunktsetzung in der Prüfung mit doppelter Gewichtung in die Gesamtnote einfloss. Ihnen stand die übliche Notenskalierung (1,0, 1,3, 1,7, 2,0 usw.) zur Verfügung. Im Durchschnitt wurde der Teil $1 \mathrm{mit}$ der Note 1,43 (Median = 1,0; Bereich: 1,0-3,0) benotet und der Teil 2 mit der Note 1,12 (Median = 1,0; Bereich: 1,0-2,0). Die Gesamtdurchschnittsnote betrug 1,19 $($ Median $=1,0$; Bereich: 1,0-2,3).

\section{Ergebnisse der Evaluation}

12 der 14 zu prüfenden Studierenden nahmen an der Evaluation teil und beantworteten den FEKaP-Pk. Zudem liegen die Evaluationsbögen pro Prüfung aus Sicht der Prüfenden (28 ausgefüllte FEKaP-P von den beiden Prüfenden aus 14 Prüfungen) sowie aus Sicht der Simulationspatientin (14 ausgefüllte FEKaP-S) vollständig vor.

\section{Ergebnisse der Prüfungskandidierenden}

In der Abbildung 1 sind die Einschätzungen des FEKaP$\mathrm{Pk}$ durch die prozentuelle Darstellung der Häufigkeiten der 5 Antwortkategorien pro Item dargestellt. Im Folgenden werden jeweils die Mittelwerte einschließlich der Standardabweichungen der wichtigsten Items zusammengefasst.

Es wird deutlich, dass das Prüfungsformat von den Prüfungskandidierenden als sehr geeignet erlebt wurde, die in den Seminaren erworbenen praktischen Fertigkeiten abzubilden (Item 4, M1 = 4,92 $\pm 0,29$ ). Hier fiel der Mittelwert höher aus als bei der Einschätzung, ob die Prüfung geeignet war, das erworbene Wissen aus den Seminaren abzubilden (Item 3, M = 4,58 $\pm 0,67$ ). Zudem wurde die Prüfung mehrheitlich als objektiv erlebt (Item $5, M=4,75 \pm 0,62$ ) und die Fragen als eindeutig und verständlich formuliert (Item 9, $M=4,67 \pm 0,49)$. Die Prüfung hat sowohl einen mittelstarken Stress (Item 7, M = 3,58 $\pm 1,16$ ) als auch recht viel Spaß (Item 8, M = 4,25 $\pm 0,87$ ) ausgelöst. Der Aufwand zur Vorbereitung der Prüfung erschien den Prüfungskandidierenden angemessen (Item 10, $M=4,67 \pm 0,49$ ). Auch der Aussage, dass "durch die Vorbereitung auf diese Prüfung das Interesse und die Begeisterung für das Fach klinische Psychologie und Psychotherapie zugenommen hat", stimmten die Studierenden mehrheitlich zu (Item 11, M = $4,58 \pm 0,62$ ). Schließlich half die Vorbereitung auf die Prüfung den meisten Prüfungskandidierenden bei der Entscheidung, ob sie die Ausbildung zum psychologischen Psychotherapeuten nach dem Studium absolvieren (Item $12, M=4,17 \pm 1,19) .5$ von 12 der Prüfungskandidierenden gaben an, bereits entschieden zu haben, die Ausbildung zum/zur psychologischen PsychotherapeutIn nach dem Studium zu beginnen (5 unklar, 2 nein).

\section{Ergebnisse der Prüfenden}

In der Abbildung 2 sind die Einschätzungen des FEKaP-P durch die prozentuelle Darstellung der Häufigkeiten der 5 Antwortkategorien pro Item abgebildet. Im Folgenden werden erneut jeweils die Mittelwerte einschließlich der Standardabweichungen der wichtigsten Items zusammengefasst.

Auch die Prüfenden gaben im Durchschnitt aller durchgeführten Prüfungen an, dass sie dieses Prüfungsformat als sehr geeignet empfanden, die in den Seminaren erworbenen praktischen Fertigkeiten abzubilden (Item A4, M = $4,93 \pm 0,26$ ). Hier fiel der Mittelwert ebenfalls höher aus als 


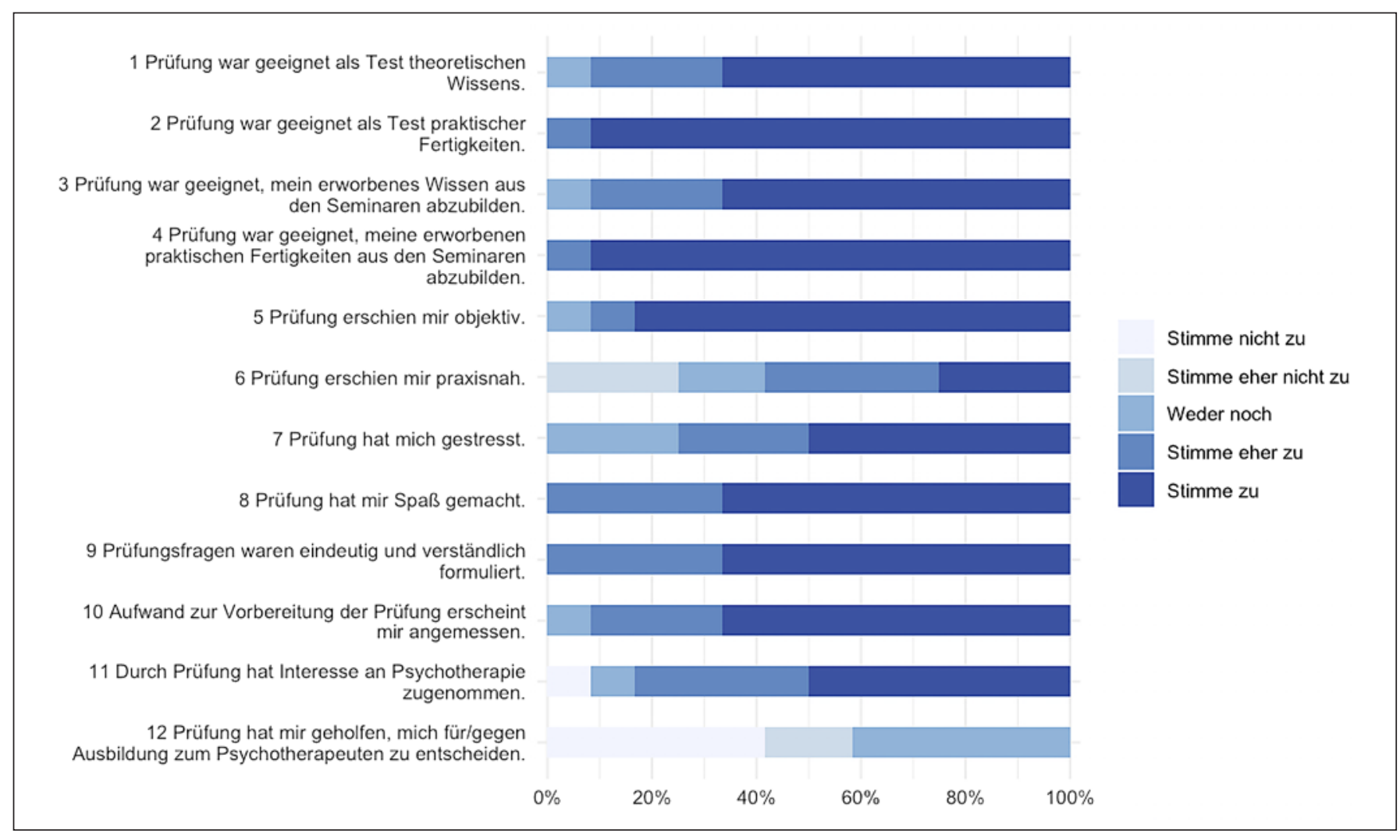

Abb. 1. Prozentuelle Darstellung der Häufigkeiten der 5 Antwortkategorien pro Item des FEKaP-Pk (Fragebogen zur Evaluation einer Kurzform der anwendungsorientierten Parcoursprüfung - Version Prüfungskandidierende), $n=12$.

bei der Frage, ob die Prüfung geeignet war, das erworbene Wissen aus den Seminaren abzubilden (Item A3, M = 4,39 $\pm 0,57$ ). Zudem erschien die Prüfung den Prüfenden vom Schwierigkeitsgrad her angemessen (Item A5, M = 4,82 \pm 0,39 ) und praxisnah (Item A6, $M=4,89 \pm 0,31$ ). Die Prüfenden stimmten den Aussagen, dass sie objektiv über die Prüfungsleistung urteilen sowie eine standardisierte Bewertung abgeben konnten, eher zu (Item P1, M = 4,36 \pm $0,56$; Item $\mathrm{P} 4, \mathrm{M}=4,43 \pm 0,50)$. Sie empfanden die Prüfung als sehr fair (Item P2, M = 4,86 $\pm 0,36$ ) und gaben an, die Prüfungsfragen eindeutig und verständlich formuliert haben zu können (Item P3, M = 4,71 $\pm 0,46$ ). Die Prüfenden gaben an, sich eher nicht gestresst (Item P5, M = 1,21 \pm 0,42 ) und von der Durchführung nicht überfordert (Item $\mathrm{P} 6, \mathrm{M}=1,07 \pm 0,26)$ gefühlt zu haben. Insgesamt gaben sie an, Spaß an der Prüfung erlebt zu haben (Item P7, M = 4,57 $\pm 0,57)$. Die Prüfungskandidierenden haben sie im Durchschnitt eher nicht nervös (Item S1, M = 2,39 $\pm 1,03$ ), eher nicht gestresst (Item S4, M =2,43 $\pm 1,20$ ) und nicht von der Prüfungssituation überfordert (Item S5, $M=1,50 \pm 0,84$ ) erlebt. Sie stimmten eher zu, dass die Prüfungskandidierenden Spaß an der Prüfung hatten (Item S6, M = 3,71 $\pm 0,90$ ) und sehr zu, dass sie an den geprüften Lehrinhalten interessiert wirkten (Item $S 7, M=4,57 \pm 0,57$ ). Insgesamt erschienen die Prüfungskandidierenden für die Arbeit als
Psychologische(r) PsychotherapeutIn den Prüfenden geeignet (Item S8, $M=4,50 \pm 0,75$ ). Die Prüfenden stimmten der Aussage zu, dass die Prüfungskandidierenden im Umgang mit der Simulationspatientin authentisch wirkten (Item M1, M = 4,54 $\pm 0,69$ ), und stimmten eher zu, dass die Prüfungskandidierenden sich auf die dargestellten Rollenspielsituationen einlassen konnten (Item M2, M = 4,46 \pm 0,79). Die Simulationspatientin schätzten sie im Umgang mit dem Prüfungskandidierenden als sehr authentisch ein (Item M3, M = 4,93 $\pm 0,26$ ) und stimmten zu, dass sie realistische Fälle gespielt hat (Item M4, M = 4,89 $\pm 0,42$ ). Schließlich stimmten sie zu, dass der Schwierigkeitsgrad der dargestellten Situation für die Prüfung angemessen (Item M5, M = 4,93 $\pm 0,26$ ) und nah am therapeutischen Alltag (Item M6, M = 4,68 $\pm 0,61$ ) war. Die mittlere Intraklassen-Korrelation zwischen den Prüfenden bezogen auf alle Items betrug $\operatorname{ICC}(C, 1)=0,88$.

\section{Ergebnisse der Simulationspatientin}

Die Abbildung 3 visualisiert schließlich die Einschätzungen des FEKaP-S durch die prozentuelle Darstellung der Häufigkeiten der 5 Antwortkategorien pro Item. Im Folgenden werden jeweils die Mittelwerte einschließlich der Standardabweichungen der wichtigsten Items zusammengefasst. 
A1 Prüfung war geeignet, theoretisches Wissen abzubilden.

A2 Prüfung war geeignet, die praktischen Fertigkeiten des Studierenden abzubilden.

A3 Prüfung war geeignet, das erworbene Wissen der

Studierenden aus den Seminaren abzubilden. A4 Prüfung war geeignet, die erworbenen praktischen Fertigkeiten der Studierenden aus den Seminaren abzubilden A5 Prüfung erschien mir vom Schwierigkeitsgrad angemessen.

A6 Prüfung erschien mir praxisnah.

P1 Ich konnte objektiv über die Prüfungsleistung urteilen.

P2 Ich konnte eine faire Prüfung durchführen.

P3 Ich konnte die Prüfungsfragen eindeutig und verständlich formulieren.

P4 Ich konnte standardisierte Bewertungen abgeben.

P5 Ich fühlte mich gestresst.

P6 Ich war mit der Durchführung überfordert.

P7 Ich hatte Spaß an der Prüfung.

S1 Studierende wirkte nervös

S2 Studierende wirkte im Umgang mit mir authentisch.

S3 Studierende wirkte gestresst

S4 Studierende wirkte mit der Prüfungssituation überfordert.

S5 Studierende schien Spaß an der Prüfung zu haben.

S6 Studierende wirkte Interessiert an den geprüften Lehrinhalten.

S7 Studierende erscheint mir für die Arbeit als Psychologische/r Psychotherapeutin geeignet. M1 Studierende wirkte im Umgang mit Simulationspatient/ authentisch.

M2 Studierende konnte sich auf die dargestellte Situation einlassen.

M3 Simulationspatient wirkte im Umgang mit dem/der Studierenden authentisch.

M4 Simulationspatient hat einen realistischen Fall gespielt.

M5 Der Schwierigkeitsgrad der dargestellten Situation war für diese Prüfung angemessen.

M6 Die dargestellte Situation war nah am klinischen Alltag.
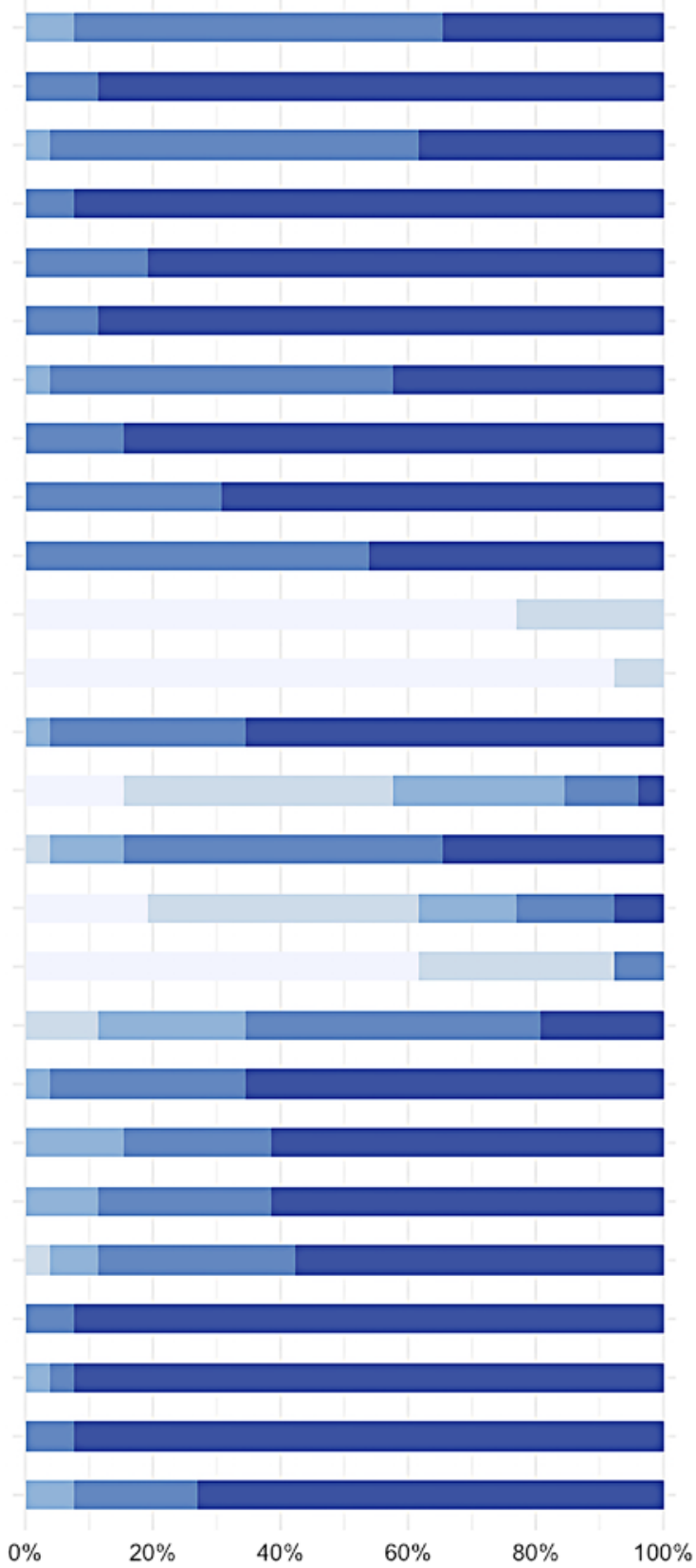

Stimme nicht zu

Stimme eher nicht zu

Weder noch

Stimme eher zu

Stimme zu

Abb. 2. Prozentuelle Darstellung der Häufigkeiten der 5 Antwortkategorien pro Item des FEKaP-P (Fragebogen zur Evaluation einer Kurzform der anwendungsorientierten Parcoursprüfung - Version Prüfende), $n=28$.

Die Simulationspatientin gab in allen durchgeführten Prüfungen an, dass sie dieses Prüfungsformat als sehr geeignet empfand, die praktischen Fertigkeiten abzubilden (Item Al, M = 5,00 $\pm 0,00$ ). Zudem erschien ihr die Prüfung eindeutig praxisnah (Item $A 2, M=5,00 \pm 0,00$ ). Sie stimmte zu, dass sie die Schwierigkeit der von ihr dargestellten Situationen als angemessen empfand (Item P1,
$M=5,00 \pm 0,00)$ und in ihren Rollen authentisch mit den Prüfungskandidierenden umgehen konnte (Item P2, M = $5,00 \pm 0,00$ ). Die Simulationspatientin gab an, sich nicht gestresst gefühlt zu haben (Item P3, $M=1,21 \pm 0,43$ ). Insgesamt schien sie Spaß in den Prüfungen erlebt zu haben (Item $\mathrm{P} 4, \mathrm{M}=4,50 \pm 0,65$ ). Sie fühlte sich auf die Prüfungen durchweg gut vorbereitet (Item P5, M = 5,00 \pm 


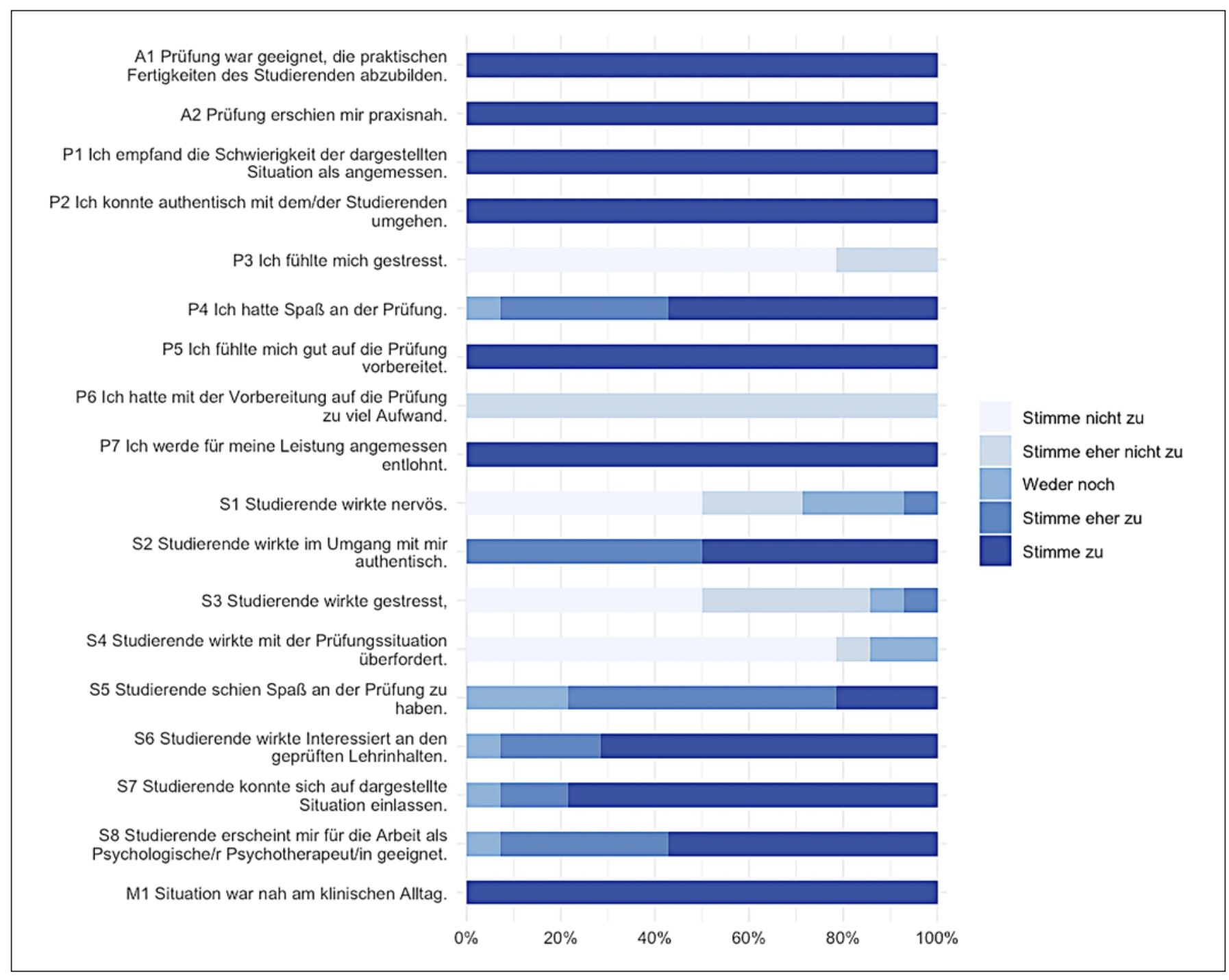

Abb. 3. Prozentuelle Darstellung der Häufigkeiten der 5 Antwortkategorien pro Item des FEKaP-S (Fragebogen zur Evaluation einer Kurzform der anwendungsorientierten Parcoursprüfung - Version Simulationspatientin oder Simulationspatient), $n=14$.

0,00) und stimmte der Einschätzung eher nicht zu, mit der Vorbereitung auf die Prüfung viel Aufwand gehabt zu haben (Item P6, $M=2,00 \pm 0,00$ ). Für ihre Leistungen fühlt sie sich angemessen entlohnt (Item P4, M = 5,00 \pm $0,00)$. Die Prüfungskandidierenden hat sie im Durchschnitt eher nicht nervös (Item S1, M = 1,86 $\pm 1,03$ ), eher nicht gestresst (Item $S 3, M=1,71 \pm 0,91$ ) und nicht von der Prüfungssituation überfordert (Item S4, M = 1,36 \pm $0,74)$ erlebt. Sie stimmte zu, dass die Prüfungskandidierenden im Umgang mit ihr authentisch wirkten (Item S2, $M=4,50 \pm 0,52)$. Sie stimmte eher zu, dass die Prüfungskandidierenden Spaß an der Prüfung hatten (Item S5, $M=4,00 \pm 0,68)$, und sehr zu, dass sie an den geprüften Lehrinhalten interessiert wirkten (Item S6, M = 4,64 \pm 0,63 ) sowie sich auf die dargestellten Situationen als Therapierende einlassen konnten (Item $S 7, M=4,71 \pm 0,61$ ). Insgesamt erschienen der Simulationspatientin die Prü- fungskandidierenden für die Arbeit als psychologische(r) PsychotherapeutIn geeignet (Item S8, M = 4,50 $\pm 0,65$ ). Schließlich stimmte die Simulationspatientin der Aussage zu, dass die dargestellten Situationen nah am therapeutischen Alltag waren (Item M1, M = 5,00 $\pm 0,00$ ).

Die wenigen Antworten auf die offenen Fragen der drei Fragebögen können im Online-Supplementmaterial 4 eingesehen werden, wobei die wichtigsten Aspekte in die folgende Diskussion integriert wurden.

\section{Diskussion und Ausblick}

In diesem Artikel wurden eine Kurzform der in der neuen PsychThApprO festgelegten anwendungsorientierten Parcoursform skizziert, die erste Durchführung beschrieben sowie die Ergebnisse eine Evaluation dargestellt. 
Vergleich zwischen der neu konzipierten Kurzform mit der in der PsychThApprO beschriebenen

Parcoursprüfung

Im Folgenden soll zunächst ein Vergleich zwischen der neu konzipierten Kurzform mit der in der PsychThApprO beschriebenen Parcoursprüfung erfolgen. Insgesamt finden sich viele Übereinstimmungen der beiden Formate: Wie im Gesetzestext beschrieben, sind in der von uns entwickelten Kurzform ebenfalls einzelne Kompetenzbereiche auf Basis strukturierter Bewertungsbögen geprüft worden. Die Prüfungsaufgaben beinhalteten zum einen eine kurze Beschreibung der Patientensituation, Angaben hinsichtlich zugelassener Hilfsmittel sowie eine Rollenbeschreibung für die/den SimulationspatientIn bezüglich der im Seminar behandelten Fälle. Hiermit gleicht die von uns konzipierte neue Prüfung den Vorgaben im Gesetz. In der Musterlösung für die Bewertung (siehe oben) waren, wie in der PschThApprO gefordert, die gewichteten Leistungsmerkmale inklusive aufgabenspezifischer Einzelkriterien enthalten, für welche wir eine höchstmögliche Punktzahl definiert haben. Des Weiteren entsprechen sich die Formate bezüglich der Schulung der SimulationspatientInnen, welche wir im Rahmen der Vorbereitung durchgeführt haben. In unserem Fall entfielen jedoch größtenteils die beschriebene Schulung sowie die Instruktionen für die Prüfenden, da diese als Lehrende der geprüften Seminare bereits mit den Kompetenzbereichen und den erwarteten Lösungen vertraut waren. Es erfolgte jedoch unter den 3 Prüfenden eine genaue Beschreibung der jeweils relevanten Seminarinhalte und entsprechenden Kompetenzbereiche.

Insgesamt kann festgehalten werden, dass die neu konzipierte Kurzform einer Parcoursprüfung weitgehend den Kriterien der in der PsychThApprO beschriebenen Parcoursprüfung entspricht, wobei der zeitliche Umfang deutlich geringer ausfiel und sich die geprüften Kompetenzbereiche inhaltlich teilweise deutlich unterscheiden. Nur die hier geprüften Kompetenzbereiche 7-9 scheinen in etwa dem in der PsychThApprO beschriebenen Kompetenzbereich 2 (therapeutische Beziehungsgestaltung) zu entsprechen, da hier die Prüfungskandidierenden ebenfalls zu zeigen haben, dass sie Probleme in der therapeutischen Beziehungsgestaltung erkennen und diesen Problemen in geeigneter Form begegnen können [vgl. Bundesgesetzblatt, 2020, $\$ 48$ Absatz 3]. Zudem erscheinen gewisse Überschneidungen zwischen dem hier geprüften Kompetenzbereich 1 und dem in der PsychThApprO vorgesehenen Bereich 5 (leitlinienorientierte Behandlungsempfehlungen), in welchem die Prüfungskandidierenden zu zeigen haben, dass sie die PatientInnen angemessen und diagnosebezogen über empfohlene Behandlungsmöglichkeiten informieren. Nicht einbezogen wurde hier die Kompetenz, dabei auch solche Behandlungsmöglichkeiten einzubeziehen, die außerhalb des eigenen Spezialisierungsbereichs liegen [vgl. Bundesgesetzblatt, 2020, $₫ 48$ Absatz 6]. Ansonsten wurden in unserer kurzen Parcoursprüfung entsprechend des bestehenden Moduls die Basistechniken der Verhaltenstherapie und moderner Psychotherapiemethoden geprüft, während in der PsychThApprO durch die 5 Stationen verfahrensübergreifende Kompetenzen der Psychotherapie adressiert werden.

Abschließend soll eine Einschätzung der Gütekriterien, welche für die medizinischen OSCE-Prüfungen formuliert wurden [vgl. Einleitung; Chenot und Erhardt, 2003], für unser Prüfungsformat erfolgen: Die geforderte Objektivität haben wir durch die entwickelten Bewertungsbögen zu sichern versucht. Die Evaluation weist jedoch auf Optimierungsbedarf hin, da die Prüfenden den Aussagen, dass sie objektiv über die Prüfungsleistung urteilen sowie eine standardisierte Bewertung abgeben konnten, im Durchschnitt nur "eher zustimmten" (statt "zustimmten"). Die zu Prüfenden gaben hingegen mehrheitlich an, die Prüfung als objektiv erlebt zu haben, wobei diese Ergebnisse unter der Limitation interpretiert werden müssen, dass Abhängigkeitsverhältnisse (evaluierende WissenschaftlerInnen sind die Lehrenden/Bewertenden) zu einer positiveren Bewertung geführt haben könnten. Die Konsistenz zwischen den Bewertungen der Prüfenden sowie das Festsetzen einer Bestehensgrenze weist auf eine hohe Reliabilität hin, welche jedoch durch die hier eröffnete Wahlmöglichkeit der Kompetenzbereiche (1-2 von 9) gemindert wird. Die durch das praktische Parcoursprüfungsformat augenscheinlich hohe Validität wird durch die Evaluation untermauert, da hier alle an der Prüfung beteiligten Personen übereinstimmend angaben, dass die Prüfung die in den Seminaren erworbenen praktischen Fertigkeiten sehr gut abbildet und die im Rollenspiel aktiven Personen (Prüfungskandidierende und Simulationspatientin) authentisch wirkten. In zukünftigen Studien sollten die drei Gütekriterien durch Evaluationen unabhängiger Wissenschaftler erfasst werden.

\section{Kritische Diskussion der Lernerfahrungen während des ersten Durchlaufs der Parcoursprüfung}

Bezüglich des ersten Prüfungsdurchlaufs sind folgende Lernerfahrungen gemacht worden: Viele strukturelle und organisatorische Aspekte konnten gut umgesetzt werden. Dabei haben sich insbesondere die schriftlichen Dokumente (speziell das Informationsblatt, vgl. Online-Supplementmaterial 1) als hilfreich für die Vorbereitung und Orientierung der Prüfungskandidierenden sowie zusätzlich zur Standardisierung für die Prüfenden und die Simulationspatientin erwiesen. Für die Simulationspatientin stellten die Lehrvideos [Barlow und Farchione, 2018; https://www.youtube.com/channel/ UC3Q9uoV1XPVzY_Q9cUgiiTg; Brakemeier, 2019] samt Erläuterungen der Seminarleiter bei der Vorbereitung auf die entsprechenden Kompetenzbereiche wert- 
volle Hilfen dar. Entsprechend dieser Erfahrung zeigen auch Studien, dass Lehrvideos nachgewiesenermaßen eine akkuratere Performance von SimulationspatientInnen gewährleisten [Schlegel et al., 2015].

Insgesamt ergab die Durchführung dieser Prüfung einen relativ hohen organisatorischen Aufwand, welcher im Voraus berücksichtigt werden sollte. Dieser ist in unserem Falle jedoch vermutlich der recht kurzfristigen Einführung des neuen Prüfungsformates (einschließlich der Erstellung der Materialien und der Fragebögen) geschuldet und könnte bei ausreichendem Vorlauf und gewonnener Routine erheblich reduziert werden. Als weiterer Aspekt wurde bei der Durchführung bezüglich des veranschlagten Zeitrahmens (insgesamt 30 min pro Prüfungskandidierenden) deutlich, dass die 5 vorgesehenen Minuten zur Nachbesprechung der Prüfenden einschließlich der Mitteilung der Note an den Prüfungskandidierenden nicht ausreichten. Aufgrund dessen kam es zu Verzögerungen im zeitlichen Ablauf der Prüfungen. Daher empfehlen wir einen längeren Zeitraum für die Nachbesprechung einschließlich der Notenmitteilung von bis zu 15 min. Dies würde auch ermöglichen, dass bei divergierenden Meinungen zur Ausführung der Rollenspiele bzw. der Notenvergabe ausreichend Zeit zur Diskussion zur Verfügung steht sowie mit Ruhe dem Prüfungskandidierenden die Notenvergabe erläutert werden kann. Damit Prüfende von der Prüfung auch langfristig profitieren, sollte es insbesondere bei schlechteren Noten ausreichend Zeit geben, auf die Defizite hinzuweisen und gleichzeitig die Stärken oder Bemühungen zu loben. Daher erscheint es empfehlenswert, diese Prüfungen alle 45 min zu terminieren.

\section{Notenvergabe}

Bei der Notenvergabe zeigte sich, dass der Durchschnitt mit 1,19 (Standardabweichung $=0,38$, Median $=$ 1, Bereich: 1-2,3) insgesamt hoch lag und wenig zwischen den Prüfungen differenzierte. Hier wäre zu überlegen, ob in folgenden Parcoursprüfungen der Schwierigkeitsgrad eventuell angepasst werden sollte. Auf Basis der zur Verfügung stehenden Materialen konnten die Prüfungskandidaten sich sehr gezielt auf die Prüfung vorbereiten. Insbesondere die Lehrvideos zu den Kompetenzbereichen 7-9 ermöglichten Imitationslernen. Um den Schwierigkeitsgrad etwas anzuheben, könnten beispielsweise die SimulationspatientInnen komplexere oder weniger aus den Seminaren bekannte Fälle spielen.

\section{Diskussion der Ergebnisse der Fragebogenevaluation}

Die Evaluation ermöglicht den Vergleich der drei Perspektiven: Alle drei Gruppen gaben nahezu gleichermassen an, dass sie das neue Prüfungsformat für geeignet hal- ten, praktische Fertigkeiten der Studierenden abzubilden und die gesamte Prüfung praxisnah, angemessen schwierig und fair empfanden.

Unterschiede wurden bei der Einschätzung im Selbsterleben der Prüfung deutlich, da sich Studierende augenscheinlich gestresster fühlten als Prüfende und die Schauspielpatientin, wobei hier auch ein Unterschied im Selbstund Fremdurteil zu beobachten ist: Die Prüfenden und die Simulationspatientin empfanden die Studierenden weniger gestresst, als sie sich selbst einstuften. Beim erlebten Spaß ist das Bild einheitlicher: Allen schien die Prüfung überwiegend $S p a ß$ gemacht $z u$ haben. Hier stimmte Selbst- und Fremdbeurteilung nahezu überein.

\section{Limitation dieser Studie}

Als wichtige Limitation ist die selektive Stichprobe (positive Consumer) anzusehen, da am neuen Prüfungsformat vermutlich überwiegend hoch motivierte und leistungsstarke Psychologiestudierende teilgenommen haben. Diese Selektion ergibt sich dadurch, dass diese Prüfung auch nach dem Sommersemester 2020 abgelegt werden kann, sodass sich in diesem Semester vermutlich vor allem jene Prüfungskandidierende angemeldet haben, welche sich das neue Format definitiv zugetraut haben. Insofern ist anzunehmen, dass die Evaluation entsprechend positiver ausgefallen ist. Des Weiteren haben sich - eventuell aus denselben Gründen - nur 2 Prüfungskandidierende für das klassische Format angemeldet, von denen beide sich aufgrund von Krankheit wieder abgemeldet hatten. Entsprechend fehlt für diese Pilotevaluation eine Kontroll- bzw. Vergleichsgruppe für einen direkten Vergleich des alten und neuen Formates. Zusätzlich ist als Limitation anzusehen, dass die evaluierenden Prüfenden das Prüfungsformat selbst konzipiert haben, weswegen ein Forscher Allegiance-Effekt zugunsten des neuen Formates nicht auszuschließen ist. Außerdem ist als Limitation zu nennen, dass der Evaluationsfragebogen (FEKaP) selbst konstruiert wurde und aufgrund der kurzfristigen Einführung nicht validiert werden konnte. Deckeneffekte weisen darauf hin, dass die Items vom Schwierigkeitsgrad zu einfach sind und nicht gut differenzieren. Sozial erwünschte Antworten sind nicht auszuschließen. Schließlich konnten durch diese Prüfung nur die Kompetenzbereiche geprüft werden, welche auch zuvor in den Seminaren behandelt wurden, weshalb psychodynamische oder systemische Kompetenzen nicht adressiert werden konnten.

\section{Ausblick}

Diese Pilotstudie weist darauf hin, dass das neue Prüfungsformat von den Beteiligten gut akzeptiert wird und sich sowohl organisatorisch gut durchführen lässt als 
auch die erlernten Fertigkeiten angemessen abbilden kann. Insbesondere der letzte Aspekt wird von den Prüfungskandidierenden, den Prüfenden und der Simulationspatientin sehr positiv bewertet.

Offen bleiben folgende Fragen, welche durch zukünftige Implementierungen von Parcoursprüfungsformaten samt Evaluationen adressiert werden sollten:

Inwieweit lassen sich die von der PsychThApprO geforderten 5 verfahrensübergreifenden Kompetenzbereiche, welche wir nicht in unserem Format adressiert haben, systematisch und standardisiert mittels SimulationspatientInnen variieren und prüfen?

Wie können die Bewertungsbögen optimiert werden, damit die Prüfenden noch objektiver und standardisierter die Prüfungsleistung beurteilen können?

Durch welche Maßnahmen kann der organisatorische Aufwand solcher Modul-Parcoursprüfungen sowie der in der PsychThApprO beschriebenen Parcoursprüfungen im Rahmen gehalten werden?

Die erste Frage weist auch auf die von vielen Instituten als Herausforderung empfundene Gewährleistung der im Gesetz geforderten Verfahrensbreite hin. Die Verfahrensbreite sollte zukünftig durch Öffnung der Lehrveranstaltungen für andere Richtlinienverfahren jenseits der Verhaltenstherapie umgesetzt werden. Bereits in dem hier beschriebenen Seminar "Moderne Ansätze der Psychotherapie" wurde psychotherapeutische Vielfalt und Breite durch die praxisorientierte Lehre von vier verschiedenen Methoden ermöglicht, wobei durch die mentalisierungsbasierte Psychotherapie auch eine explizit aus der psychodynamischen Tradition stammende Methode einbezogen wurde.

Obwohl einige organisatorische Punkte noch zu klären sind, konnten anhand dieser Kurzform einer Parcoursprüfung wichtige erste Erfahrungen hinsichtlich der Implementierung eines anwendungsorientierten Formates gewonnen werden. Da sich insbesondere eine ausreichende Vorbereitung von Prüfungsunterlagen, Bewertungsbögen sowie die umfassende Schulung der Simulationspatientin mittels Lehrvideos sowie durch die Prüfenden sich bewährt haben, werden diese Aspekte auch für zukünftige Parcoursprüfungen von uns empfohlen. Außerdem zeigte sich durch die überwiegend positiven Bewertungen aller drei Gruppen, dass solche Formate eine überzeugende und attraktive Alternative $\mathrm{zu}$ dem bisherigen klassischen Prüfungsmodul darstellen.
Zukünftige Evaluationen derartiger Parcoursprüfungen - von unabhängigen Wissenschaftlern durchgeführt werden dazu beitragen, das Format weiter zu optimieren und dabei insbesondere auch die drei in den OSCE-Prüfungen etablierten Gütekriterien zu adressieren.

Insgesamt können die Ergebnisse dieser Pilotstudie als guter Indikator dafür angesehen werden, dass die nun kommende Herausforderung - eine anwendungsorientierte Parcoursprüfung für das neue Approbationsstudium zu konzipieren und Varianten dieser Prüfungsform in die neuen Studiengänge zu integrieren - von den psychologischen Instituten erfolgreich bewältigt werden kann.

\section{Acknowledgment}

Unser herzlichster Dank gilt den Prüfungskandidierenden, welche den Mut aufbrachten, diese neue Prüfungsform zu wählen und an der Evaluation teilzunehmen. Zudem danken wir Philipp Herzog, der das praxisrelevante Seminar zu Zwangsstörungen gegeben hat, sowie Doris Kehl, die die Dateneingabe übernommen hat. Alfons Hamm und seinem Team gebührt unser aufrichtiger Dank für die bisherige Ausbildung der Studierenden im Fach klinische Psychologie und Psychotherapie an der Universität Greifswald.

\section{Statement of Ethics}

Für diese Übersichtsarbeit war keine Prüfung durch eine Ethikkommission nötig.

\section{Disclosure Statement}

Die Autoren erklären, dass keinerlei Interessenkonflikte oder finanzielle Förderungen im Hinblick auf die vorliegende Arbeit bestehen.

\section{Author Contributions}

Eva-Lotta Brakemeier schrieb die erste Version des Manuskripts. Tim Kaiser führte die Auswertungen der Evaluation durch und las das gesamte Manuskript kritisch gegen. Kristin Pilz ergänzte das Manuskript insbesondere auf Basis ihrer langjährigen Erfahrung mit OSCE-Prüfungen und aus Sicht der Simulationspatientin. Jan Richter trug zum finalen Artikel durch Ergänzungen und Veränderungen basierend auf seiner langjährigen Lehr- und Prüfungserfahrung bei.

\section{Literatur}

Barlow DH, Farchione TJ, editors. Applications of the unified protocol for transdiagnostic treatment of emotional disorders. New York: Oxford University Press; 2018.

Brakemeier EL. Schwierige Situationen in der modernen Psychotherapie. CBASP, DBT,
MBT und Schematherapie. Beltz VideoLearning. Weinheim: Beltz; 2019.

Bundesgesetzblatt (Jahrgang 2020). Teil I Nr. 11, ausgegeben zu Bonn am 12. März 2020.

Bundesministerium für Gesundheit: Entwurf einer Approbationsordnung für Psycho- therapeutinnen und Psychotherapeuten, 2019.

Chenot JF, Ehrhardt M. Objective structured clinical examination (OSCE) in der medizinischen Ausbildung: Eine Alternative zur Klausur. ZFA (Stuttgart). 2003;79(09):437-42. 
Chisnall B, Vince T, Hall S, Tribe R. Evaluation of outcomes of a formative objective structured clinical examination for second-year UK medical students. Int J Med Educ. 2015 Jun;6:76-83.

Harden RM, Stevenson M, Downie WW, Wilson GM. Assessment of clinical competence using objective structured examination. BMJ. 1975 Feb;1(5955):447-51.

Kanfer FH, Reinecker H, Schmelzer D. Selbstmanagement-Therapie. Berlin, Heidelberg, New York: Springer-Verlag; 2012. https://doi. org/10.1007/978-3-642-19366-8.

Schlegel C, Bonvin R, Rethans JJ, van der Vleuten C. The use of video in standardized patient training to improve portrayal accuracy: A randomized post-test control group study. Med Teach. 2015 Aug;37(8):730-7.

Turner JL, Dankoski ME. Objective structured clinical exams: a critical review. Fam Med. 2008 Sep;40(8):574-8.

Vaidya NA, Ramchandani D. Psychiatry clerkship objective structured clinical examination is here to stay. Acad Psychiatry. 2008 MayJun;32(3):177-9.

Wahl-Kordon A, Zurowski B, Wahl K, Hohagen F. Zwangsstörungen. In: Berger $\mathrm{M}$, editor. Psychische Erkrankungen. München: Urban \& Fischer; 2019. p. 483-500.e2.

Wilhelm M, Rief W, Haberkamp A, von Blankenburg P, Glombievsky JA. Studium zur Psychotherapie-Approbation als Herausforderung und Chance: Praktische Hochschullehrerkonzepte in der klinischen Psychologie und Psychotherapie. Verhaltenstherapie. Im Druck. 